\title{
Induction of Cytotoxic T-Lymphocytes and Antitumor Activity by a Liposomal Lipopeptide Vaccine
}

\author{
Weihsu Chen ${ }^{\dagger} \ddagger$ and Leaf Huang ${ }^{*}, \dagger$ \\ $\dagger$ Division of Molecular Pharmaceutics, School of Pharmacy, UniVersity of North Carolina at Chapel Hill, \\ Chapel Hill, North Carolina 27599 \\ $\$$ Department of Pharmaceutical Sciences, School of Pharmacy, UniVersity of Pittsburgh, Pittsburgh, \\ PennsylVania 15213
}

\section{Abstract}

We have previously described a simple yet effective liposome-based therapeutic vaccine, DOTAP/ E7, which contains only two molecules, the cationic lipid DOTAP and a peptide antigen derived from the E7 oncoprotein of human papillomavirus (HPV) type 16. In the current report, we have improved the vaccine formulation by incorporation of E7-lipopeptide instead of the water-soluble native E7 peptide into the DOTAP liposome. The lipopeptide consists of an N-terminal $\alpha$ - or $\epsilon$ palmitoyl lysine connected to the E7 peptide via a dipeptide Ser-Ser linker. The DOTAP/E7lipopeptide vaccine exhibited an enhanced functional antigen-specific $\mathrm{CD} 8^{+} \mathrm{T}$ lymphocyte response in vivo compared to the previous DOTAP/E7 formulation. More importantly, the cytotoxic T cells induced by the DOTAP/E7-lipopeptide vaccine could efficiently eliminate an existing HPV positive TC-1 tumor. The antitumor activity of lipopeptide formulated in DOTAP liposome was more than twice as potent as that of native E7, likely owing to the increased peptide entrapment efficiency in the liposomal complex. Our results also showed that it is essential to have the dipeptide spacer sequence between E7 peptide and the attached fatty acid to achieve a full immune response. Overall, the improved DOTAP/E7-lipopeptide vaccine described herein showed a significantly enhanced therapeutic effect for the treatment of a cervical cancer model.

\section{Keywords}

Cationic liposome; lipopeptide; vaccine; cancer immunotherapy; cervical cancer

\section{Introduction}

Liposomes have been extensively used for delivering small molecular weight drugs, ${ }^{1}$ plasmid DNA, ${ }^{2}$ oligonucleotides, ${ }^{3}$ proteins,,${ }^{4,5}$ and peptides. ${ }^{6}$ From a safety standpoint, a liposomal vehicle as a nonviral vaccine carrier has been regarded as a preferable strategy compared to traditional immunizations using live attenuated vaccines or viral vectors such as vaccinia or influenza virus. ${ }^{7,8}$ Recent studies from our group have led to the development of a simple yet effective peptide-based liposomal vaccine, DOTAP/E7 complex, which consists of only two molecules, the cationic lipid DOTAP and a peptide antigen which is derived from E7 oncoprotein of human papillomavirus (HPV) type 16. The DOTAP/E7 formulation was able

\footnotetext{
(C) 2008 American Chemical Society

* To whom correspondence should be addressed. Mailing address: 2316 Kerr Hall, CB\# 7360, Division of Molecular Pharmaceutics, School of Pharmacy, University of North Carolina at Chapel Hill, Chapel Hill, NC 27599. Tel: 919-843-0736. Fax: 919-966-0197. Email address: leafh@unc.edu.
} 
to induce both preventative and therapeutic antitumor effects against HPV positive TC-1 tumor in a mouse model. Our results have demonstrated for the first time that the cationic liposome alone not only plays the role of a carrier to deliver the peptide antigen to the antigen presenting cells (APC) but also serves as a potent vaccine adjuvant to stimulate immune responses and initiate DC-T cell interactions. 9,10

There has been considerable interest in developing lipopeptide formulations for both humoral and cellular immune response. This is in contrast to the native synthetic peptides which are poorly immunogenic. Peptides linked with monoor multipalmitic acid are capable of producing an antigen-specific cytotoxic T lymphocyte response against infectious diseases such as HIV, $11,12 \mathrm{HBV}, 13$ and malaria, ${ }^{14}$ and several clinical trials are investigating this effect. ${ }^{15,16}$ However, lipopeptides have limited solubility and require a means for solubilization. Since liposome is a good carrier for lipophilic drugs, we investigated the possibility of formulating a lipopeptide antigen in the DOTAP liposomes. Our results showed significantly improved immunogenic activity of the lipopeptide as compared to the original E7 peptide. We have also evaluated the antitumor activity of the E7-lipopeptide in DOTAP liposome in the TC-1 cervical cancer model in mice.

\section{Materials and Methods}

\section{Lipids, Reagents, and Murine Tumor Cell Lines.}

DOTAP was purchased from Avanti Polar Lipids, Inc. (Alabaster, AL). Murine TC-1 cells were kindly provided by Dr. T. C. Wu at Johns Hopkins University (Baltimore, MD). TC-1 cells are C57BL/6 mouse lung epithelial cells transformed with HPV 16 E6 and E7 oncogenes and the activated H-ras. RMA-S, a mouse lymphoma cell line, was kindly provided by Dr. J. Frelinger at the University of North Carolina at Chapel Hill. Both $\mathrm{H}-2^{\mathrm{b}}$ murine tumor cell lines were maintained in RPMI-1640 medium (Invitrogen, Carlsbad, CA) supplemented with 10\% fetal bovine serum, 100 units/mL of penicillin, and $100 \mu \mathrm{g} / \mathrm{mL}$ of streptomycin (Invitrogen).

\section{Peptide Synthesis}

Peptides and lipopeptides (Table 1) containing an $\mathrm{H}-2 \mathrm{D}^{\mathrm{b}}$-restricted CTL epitope (amino acid 49-57, RAHYNIVTF) derived from HPV 16 E7 protein were synthesized and purified at the Molecular Medicine Institute Peptide Synthesis Facility at the University of Pittsburgh. Briefly, lipopeptides used in this study were generated by elongating the N-terminal of E7 peptide with a linker peptide Lys-Ser-Ser (KSS), and a palmitic acid was then attached to the $\alpha$-or $\epsilon$-amino group of the Lys residue. An unlipidated version of KSS-elongated E7 peptide (abbreviated KSS-E7) or a palmitoylated E7 peptide (fatty acid attached to N-terminal Arg residue without a spacer sequence; abbreviated PA-E7) was used as control.

\section{Preparation and Characterization of DOTAP/Lipopeptides}

Cell culture grade water (Cambrex, Walkersville, MD) was used in all liposome preparations. Lipopeptide was mixed with DOTAP in $\mathrm{CHCl}_{3} / \mathrm{MeOH}$ (1:1 mixture) followed by evaporation under a steady stream of dry nitrogen gas. Traces of organic solvent were removed by vacuum desiccation overnight. Lipopeptide/lipid films were hydrated by adding water and incubated at room temperature for $12 \mathrm{~h}$. The suspensions were sonicated in a bath type sonicator for 10 min followed by extrusion (Hamilton Co., Reno, NV) through 400, 200, and $100 \mathrm{~nm}$ polycarbonate membrane (Nuclepore, Pleasanton, CA) and were stored at $4{ }^{\circ} \mathrm{C}$ before use. For preparation of DOTAP/E7 or DOTAP/KSS-E7, DOTAP lipid film was hydrated in an aqueous solution containing the water-soluble peptide. The unincorporated E7 peptides were not removed from the liposome before they were injected into animals. 
Peptide encapsulation efficiency was determined by the percentage of the liposome-bound peptide using LavaPep peptide quantification kit (Fluorotechnics, Sydney, Australia). Since unincorporated lipopeptide aggregated and could not pass the exclusion filter, the incorporated lipopeptide was measured as the amount associated with the extruded liposomes. The liposomes were dissolved in $1 \%$ SDS, and the amount of peptide was measured as described above. The data were reported as the mean $\pm \mathrm{SD}(n=3)$. For water-soluble peptides such as native E7 and KSS-E7, the unbound peptide was separated from the complex by a Microcon centrifugal filtration device (Millipore, Bedford, MA). ${ }^{9}$ The concentration of the unbound peptide was measured using LavaPep according to the manufacturer's instructions. The efficiency of encapsulation was determined as (100\% - \% unbound peptide) and was reported as the mean $\pm \mathrm{SD}(n=3)$. The particle size and the $\zeta$ potential of the liposomal complexes were measured following the manufacturer's instructions using a submicron particle sizer (NICOMP particle sizing systems, Santa Barbra, CA) and a ZetaPlus (Brookhaven Instruments, Corp., Holtsville, NY), respectively.

\section{Mice and Immunizations}

All work performed on animals was in accordance with and approved by our institutional IACUC. C57BL/6 female mice, 6-7 weeks old, were purchased from Charles River Laboratories (Wilmington, MA) and were used in all animal studies. Subcutaneous (s.c.) tumors were established by injecting TC- 1 cells $\left(10^{5}\right.$ cells) into the hair-trimmed flank of the mouse on day 0 . Mice were s.c. treated with a liposomal formulation comprising a constant dose of DOTAP lipid $100 \mathrm{nmol}$ and a varied amount of peptide at the other side of flank on day 6 unless otherwise stated. The size of the tumor was measured using a caliper two or three times a week. Tumor size was determined by multiplying the two largest dimensions of the tumor.

\section{Analysis of In Vivo CTL Response}

In vivo $\mathrm{CTL}$ activity of $\mathrm{E} 7$-specific cytotoxic $\mathrm{T}$ cells was enumerated according to the protocol of Byers et al. ${ }^{17}$ with minor modifications. In brief, spleen cells from syngenic mice were RBC lysed followed by pulsing with $10 \mu \mathrm{M}$ E7 peptide or without peptide in complete medium for $1 \mathrm{~h}$ at $37^{\circ} \mathrm{C}$. Both spleen cell populations were stained with equal amount of $2 \mu \mathrm{M} \mathrm{PKH}-26$ (Sigma-Aldrich) according to the manufacturer's instructions. The peptide pulsed and unpulsed populations were loaded with 4 and $0.4 \mu \mathrm{M}$ CFSE (Molecular Probes), respectively, at $37^{\circ} \mathrm{C}$ for $15 \mathrm{~min}$. The two cell populations were mixed together (1:1) for tail vein injection to the control or the immunized mice $\left(10^{7}\right.$ cells per mouse). At $16 \mathrm{~h}$ after injection, spleen cells from the recipient mice were isolated, and single cell suspensions were prepared prior to flow cytometric analysis. The number of CFSE ${ }^{\text {high }}$ and CFSE $^{\text {low }}$ populations were determined, and the in vivo E7 specific lysis percentage was enumerated according to a published equation. ${ }^{17}$

\section{Intracellular Cytokine Staining}

All antimouse antibodies used for flow cytometric analysis were purchased from BD Pharmingen (San Diego, CA) or eBioscience, Inc. (San Diego, CA). For the measurement of IFN- $\gamma$ producing $\mathrm{CD} 8^{+} \mathrm{T}$ cells, spleen cells were isolated from the control or the immunized mice 7-10 days after the last immunization. Spleen cells $\left(2 \times 10^{6}\right)$ were incubated with $5 \mu \mathrm{g} /$ $\mathrm{mL}$ of E7 peptide or without peptide for $6 \mathrm{~h}$ in the presence of $1 \mu \mathrm{L} / \mathrm{mL}$ of GolgiPlug (BD Pharmingen). After being washed with FACS buffer, cells were stained directly with anti-CD8a (53-6.7) on ice for $30 \mathrm{~min}$. Prior to staining with anti-IFN- $\gamma \mathrm{mAb}$ (XMG1.2), cells were fixed and permeabilized using the Cytofix/Cytoperm kit (BD Pharmingen) according to the manufacturer's instructions. Cells were finally resuspended in $300 \mu \mathrm{L}$ of stain buffer and analyzed using a flow cytometer. 


\section{MHC Class I Binding Assay}

RMA-S cells at a concentration of $5 \times 10^{5}$ cells $/ \mathrm{mL}$ were incubated overnight at $27{ }^{\circ} \mathrm{C}$ with native E7 or KSS-E7 peptide $(10 \mu \mathrm{M})$. Cells incubated with medium were used as a control. Cells were then transferred to $37^{\circ} \mathrm{C}$ and incubated for $2 \mathrm{~h}$. After washes, cells were stained with fluorescently conjugated mAbs against $\mathrm{H}-2 \mathrm{D}^{\mathrm{b}}$ or $\mathrm{H}-2 \mathrm{~K}^{\mathrm{b}}$ molecules on the cell surface prior to flow cytometry analysis.

\section{Statistical Analysis}

Data were analyzed statistically using a one-way ANOVA and a two-tailed Student's $t$ test. Differences in data were considered statistically significant when the $p$ value was less than 0.05 .

\section{Results}

\section{Encapsulation Efficiency of E7-Lipopeptide in the Cationic DOTAP Liposome}

In order to improve the efficiency of the DOTAP/E7 therapeutic vaccine, one of the strategies was to increase the incorporation of the antigen in the liposome by utilizing lipopeptides. The lipopeptides used in this work are described in Table 1. The particle sizes of DOTAP/E7lipopeptide were not significantly changed compared to that of the DOTAP/E7, which was $102.6 \pm 20.5 \mathrm{~nm}$ in diameter. Also, the $\zeta$ potential of DOTAP/E7-lipopeptide particles was similar to that of DOTAP/E7 ( $44.5 \pm 6.8 \mathrm{mV})$, indicating that the particle remains positively charged after the water-soluble peptide is changed to a lipopeptide. However, significant changes were found in the peptide entrapment efficiency in the liposome. When peptide loading was $2.5 \mathrm{~mol} \%$ of the total lipid, the entrapment efficiency of E7-lipopeptides (i.e., $\alpha$ - or $\epsilon$ PAKSS-E7) within DOTAP liposome reached up to $90 \%$ compared to $\sim 25 \%$ incorporation of the water-soluble native E7 and KSS-E7 peptides. As shown in Figure 1, varying amounts of $\epsilon$ PA-KSS-E7 lipopeptide (0.16 to $10 \mathrm{~mol} \%$ ) were dissolved with DOTAP lipid of $100 \mathrm{nmol}$ in an organic solvent, and the incorporation rate was measured following liposome preparation. For all lipopeptide complexes, the entrapment efficiency decreased with increasing lipopeptide loading. The incorporation rate was nearly $95 \%$ when lipopeptide concentration did not exceed $2.5 \mathrm{~mol} \%$. When the peptide loading was increased to $5 \mathrm{~mol} \%$, approximately $70-75 \%$ entrapment efficiency was measured. Furthermore, less than $60 \%$ of lipopeptide was incorporated into the liposomes when lipopeptide was loaded at $10 \mathrm{~mol} \%$. The loss of lipopeptide at higher loading ratio could be mostly recovered from the polycarbonate membrane which was used during extrusion (data not shown). These results demonstrate that lipopeptides exhibit superior entrapment efficiency compared to the native E7 peptide. However, the incorporation rate decreases with the increasing loading of lipopeptide; it exceeded the solubility limit at the high loading ratios.

\section{Antitumor Activity Was Enhanced by Incorporation of E7-Lipopeptide in the DOTAP Liposome}

Previous studies in our group have shown that a MHC class I peptide epitope derived from HPV (type 16) E7 protein encapsulated in cationic DOTAP liposomes can generate a therapeutic effect against the HPV16 E7 positive tumor cell line, TC-1.9,10 To test whether the antitumor activity is enhanced by use of the lipopeptide, a series of E7-lipopeptides (Table 1) were synthesized and formulated in the DOTAP liposomes as described. As shown in Figure 2, TC-1 tumor-bearing mice were given a single-dose treatment on day 6 with DOTAP/E7 (containing 10 or $5 \mathrm{nmol}$ of peptide) or DOTAP/E7-lipopeptide formulations containing $5 \mathrm{nmol}$ of the antigen. DOTAP/E7 with $5 \mathrm{nmol}$ of peptide (suboptimal dose) did not show a significant antitumor activity. On the contrary, both of the E7-lipopeptides ( $\alpha$ - or $\epsilon$-PA-KSS-E7) at $5 \mathrm{nmol}$ of antigen, when formulated in DOTAP liposome, showed a significantly enhanced therapeutic 
effect $(* * p<0.01)$ compared to the native $\mathrm{E} 7$ of $5 \mathrm{nmol}$. The antitumor activity elicited by the lipopeptides was similar to that of DOTAP/E7 at $10 \mathrm{nmol}$ (i.e., optimal peptide dose). DOTAP liposome alone did not show antitumor activity. PA-E7, which is a palmitoylated E7 peptide (without the KSS spacer), when formulated in DOTAP liposome failed to show an enhanced antitumor activity as seen in other lipopeptide formulations, likely owing to the epitope being hidden by directly attaching a fatty acid to the peptide. In addition, an unlipidated version of KSS-elongated E7 peptide (KSS-E7) exhibited a larger effect $(* p<0.05)$ in tumor suppression than the native E7. Under these conditions, E7-lipopeptides formulated in DOTAP liposome showed an enhanced antitumor activity compared to the original DOTAP/E7 formulation.

\section{In Vivo Cytotoxic T-Lymphocytes Were Elicited by DOTAP/E7-Lipopeptide Formulation}

It is important to evaluate whether a candidate cancer vaccine would be suitable for inducing a cytotoxic T-lymphocyte (CTL) response since the primary CTL is important for eradicating the growth of tumor cells while the memory CTL is essential for preventing cancer recurrences.

18,19 To avoid the possible overestimation of CTL function due to in Vitro restimulation and expansion process, we evaluated CTL induced in vivo by DOTAP/E7 or DOTAP/E7lipopeptide vaccine (Figure 3A). As described in the Materials and Methods, a mixture containing equal amounts of E7-pulsed CFSE ${ }^{\text {high }}$ and un-pulsed CFSE ${ }^{\text {low }}$ spleen cells from a syngenic donor was i.v. injected into mice at 7 days after the last immunization. The specific lysis of E7-pulsed cells was analyzed by flow cytometry at $16 \mathrm{~h}$ after the adoptive transfer. The mice immunized with DOTAP/E7 antigen $(10 \mathrm{nmol})$ could generate a superior E7-specific killing compared to those immunized with lower amounts of antigen DOTAP/E7 (5 nmol). In contrast to the native E7 formulation, mice receiving $5 \mathrm{nmol}$ of $\alpha$ - and $\epsilon$ PA-KSS-E7 but not PA-E7 formulated in DOTAP liposome efficiently eliminated about $80 \%$ of E7-pulsed targets (Figure 3B). In addition, the mice receiving DOTAP/KSS-E7, were also capable of killing 60\% of in vivo targets, while the mice receiving DOTAP alone did not generate a noticeable E7specific CTL response. The results suggested that E7-lipopeptides formulated in DOTAP liposome elicited an improved in vivo CTL response in than the native E7 peptide.

\section{IFN- $y$ Production from Functional CD8+ T Cells Was Induced by DOTAP/E7-Lipopeptide}

To assess the epitope-specific immune response induced by DOTAP/E7-lipopeptide vaccination, IFN- $\gamma$ producing $\mathrm{CD} 8^{+} \mathrm{T}$ cells were analyzed (Figure 4). Spleen cells from control or immunized mice were isolated at 1 week after the final immunization. After stimulation with $5 \mu \mathrm{g} / \mathrm{mL}$ of E7 peptide (Figure $4 \mathrm{~A}$, bottom) or without peptide (Figure 4A, top), intracellular staining for IFN- $\gamma$ was performed. The numbers shown in the dot plots represent the percentage of $\mathrm{CD} 8$ and IFN- $\gamma$ double-positive cells within the $\mathrm{CD} 8^{+}$population. As depicted in Figure 4B, the numbers of IFN- $\gamma$ producing $\mathrm{CD} 8^{+}$cells were significantly higher in mice that received $5 \mathrm{nmol}$ of $\epsilon$-PA-KSS-E7 formulated in the DOTAP liposomes than those of both 10 and $5 \mathrm{nmol}$ of the native E7 formulation $(* * p<0.01$ ). Again, KSS-E7 showed a superior result compared to the native $\mathrm{E} 7$ at the equal antigen amount. The IFN- $\gamma$ production by the $\mathrm{CD}^{+}$cells was in an E7-specific manner, as the unpulsed cells showed only background level of the cytokine. These results show that incorporation of $\alpha$ - or $\epsilon$ PA-KSS-E7 lipopeptides into DOTAP liposomes clearly enhanced the amount of IFN- $\gamma$ producing $\mathrm{CD}^{+} \mathrm{T}$ lymphocytes in the lymphoid organ.

\section{H-2D ${ }^{b}$ Was Up-Regulated by KSS-E7 Using RMA-S Binding Assay}

Both native E7 and KSS-E7 are water-soluble peptides that reach similar entrapment efficiency in liposome; KSS-E7 appears to have better immunogenicity compared to the native E7. To elucidate the mechanism by which KSS-E7 peptide exhibited antigen activity superior to that of the native E7, the binding of MHC class I molecules by E7 and KSS-E7 peptide was investigated. E7 peptide (a.a. 49-57) is a known epitope restricted to $\mathrm{H}-2 \mathrm{D}^{\mathrm{b}}, 20$ and it up- 
regulated more than 4-fold of $\mathrm{H}-2 \mathrm{D}^{\mathrm{b}}$ molecules on RMA-S cells compared to the control (Figure 5). Intriguingly, an 8-fold increase in the mean fluorescence was observed for KSSE7 peptide. No up-regulation of $\mathrm{H}-2 \mathrm{~K}^{\mathrm{b}}$ molecules was detected on RMA-S cells after incubation with either E7 or KSS-E7 peptide. The results suggest that the KSS-E7 slowed down the internalization of the MHC class I molecules. It is also possible that the KSS-E7 has an improved binding affinity for $\mathrm{H}-2 \mathrm{D}^{\mathrm{b}}$ molecules, or an reduced degradation, than the native $\mathrm{E} 7$ peptide which may lead to an overall superior antitumor activity when formulated in the DOTAP liposomes.

\section{Potent Antitumor Activity Was Induced by DOTAP/E7-Lipopeptide Vaccine}

To further study the potency of the DOTAP/E7-lipopeptide vaccine against the existing tumor, the varied doses of $\epsilon$ PA-KSS-E7 (i.e., 1.25-10 nmol per injection) were investigated (Figure $6 \mathrm{~A})$. The tumor-bearing mice that received one single treatment of DOTAP/ $\epsilon$ PA-KSS-E7 with 5 or $10 \mathrm{nmol}$ of antigen could induce full tumor growth inhibition $(* * p<0.01)$ compared to the partial tumor growth inhibition in those injected with DOTAP/E7 $(5 \mathrm{nmol})$. The formulation still showed a partial antitumor effect $\left({ }^{*} p<0.05\right)$ when the lipopeptide amount was decreased to as low as $1.25 \mathrm{nmol}$. To evaluate whether the improved vaccine could also be effective in the treatment of TC- 1 tumor of larger sizes, the treatment was delayed until day 10 instead of day 6 (Figure 6B). Whereas both DOTAP/E7 $(10 \mathrm{nmol})$ and DOTAP/ $\epsilon$ PA-KSSE7 $(5 \mathrm{nmol})$ vaccines given on day 6 showed an effective antitumor activity, mice receiving the lipopeptide formulation on day 10 exhibited a superior effect on tumor inhibition than the native E7 peptide given at the same day $\left({ }^{*} p<0.05\right)$. Overall, the DOTAP/E7-lipopeptide vaccine described in this work was capable of generating efficient antitumor activity even at the low antigen dose compared to the original DOTAP/E7 formulation. Moreover, the improved vaccine activity also demonstrated growth inhibition of larger tumors.

\section{Discussion}

Development of safe and effective therapeutic cancer vaccines for human use remains an urgent and unmet medical need. In this report, we demonstrate an improved liposomal lipopeptide vaccine for the treatment of HPV positive tumors. By linking to a monopalmitic acid to KSSelongated E7 peptide (at either $\alpha$ or $\epsilon$ position), the peptide encapsulation efficiency within liposomes was higher than that of the unmodified E7 peptide. The DOTAP/E7-lipopeptide vaccine induced an overall enhancement in generating antigen-specific CTL for eradicating HPV positive TC-1 cells (Figure 3 ). When a reduced amount ( $5 \mathrm{nmol}$ or less) of peptide dose was given to the tumor-bearing mice, DOTAP/E7-lipopeptide exhibited a superior antitumor activity compared to the original DOTAP/E7 formulation at a full dose $(10 \mathrm{nmol})$ (Figures 2 and 6A). Furthermore, the therapeutic effect of DOTAP/E7-lipopeptide was also effective for suppression of tumor growth in later stages of tumor progression, suggesting applications in progressed cancer treatments (Figure 6B).

The enhanced antigenicity and antitumor activity of the lipopeptide were correlated with the enhanced encapsulation of the lipopeptide in the liposomes. As shown in Table 1, the entrapment efficiency of lipopeptide reached to $90 \%$ when the peptide loading is $2.5 \mathrm{~mol} \%$ among total lipid, whereas the native water soluble E7 peptide only incorporated into the liposome at about $25 \%$. Similar enhanced liposome encapsulation of lipopeptides has also been reported. ${ }^{21-23}$ In order for the peptide to be presented by the MHC class I pathway, the peptide has to enter the cytoplasm of the APC. Cationic liposomes deliver the encapsulated, but not free, peptide into the APCs, allowing the peptide to be released and later presented in the MHC class I pathway. Thus, the significantly higher the encapsulation of the peptide would allow for increased peptide delivery and therefore, higher antigenicity, can be expected. However, there is an exception to the rule. 
Our data indicate that the lipopeptide PA-E7 without the spacer sequence Lys-Ser-Ser achieved of high degree of liposome encapsulation but did not show an enhanced antitumor activity compared to those with the spacer amino acids. The phenomenon was likely a result of some structural constraints where the conjugated fatty acid may cover the CTL epitope, thereby minimizing its recognition. Verheul et al. have also shown that the amino acid spacer sequences influence the immunological properties of the lipopeptide conjugates. ${ }^{24}$ In addition, to enter the endosomal pathway as exogenous soluble protein and peptides, lipopeptides must be processed to be free peptide again once entering into the cytoplasm of the APC. By adding a linker sequences, it may prevent the CTL epitope from being altered during the processing of the lipopeptide in the cytosol. Several additional spacer amino acids have been investigated thus far including CSS and CSKKK, ${ }^{25,26}$ and they showed a stronger immunogenicity than those without a spacer sequence.

The KSS-E7 peptide formulated in DOTAP appears to be a better vaccine formulation than the native E7 peptide as demonstrated by the tumor growth inhibition and increased antigencity, although their liposome encapsulation efficiencies were comparable. Interestingly, we found that mice receiving DOTAP/KSS-E7 generated an increased functional CTL response (Figure 3 and 4) as well as an enhanced antitumor activity (Figure 2) compared with the native E7. It is known that the capacity of a peptide to bind and stabilize MHC class I molecules is directly correlated with its ability to induce specific CTL responses. ${ }^{20,27}$ Our results tempted us to study the MHC class I binding affinity of the native and the KSS-elongated E7 peptides. RMA$S$ cell line was originally selected from mutated cells on the basis of low cell surface expression of MHC class I molecules and inability to present endogenous antigens. The synthesis of class I molecule heavy and light chains is normal; however, the mutant cells undergoes various protein degradation routes at $37^{\circ} \mathrm{C}$, resulting in a decreased number of MHC on the surface of RMA-S cells. At $27^{\circ} \mathrm{C}$, the breakdown of MHC molecules is slow, and $\mathrm{H}-2 \mathrm{D}^{\mathrm{b}}$ or $\mathrm{H}-2 \mathrm{~K}^{\mathrm{b}}$ molecules are able to appear on the cell surface at the reduced temperature. ${ }^{28}$ The labile class I molecules at $37{ }^{\circ} \mathrm{C}$ can be stabilized by exposing cells to peptides which interact with $\mathrm{H}-2 \mathrm{D}^{\mathrm{b}}$ or $\mathrm{H}-2 \mathrm{~K}^{\mathrm{b}}$. Although the detailed mechanism is not known, our results shown in Figure 5 indicated that KSS-E7 bound with a higher affinity with $\mathrm{H}-2 \mathrm{D}^{\mathrm{b}}$, but not $\mathrm{H}-2 \mathrm{~K}^{\mathrm{b}}$, molecules than the native E7 peptide. However, we cannot rule out the possibility that KSS-E7 may slow down the internalization of the MHC class I molecules. Thus, the enhanced antigenicity of $\alpha$ and $\epsilon$ PA-KSS-E7 lipopeptides was likely due to both an enhanced liposome encapsulation and an elevated biding affinity with the $\mathrm{H}-2 \mathrm{D}^{\mathrm{b}}$ molecule for antigen presentation.

In summary, the results from this report demonstrate that the improved formulation, DOTAP/ E7-lipopeptide, is a potential therapeutic vaccine for the treatment of HPV-positive tumors. The vaccine described here maintains the benefits of simplicity and safety from its original version, DOTAP/E7, which contains only two molecules, and the lipopeptide formulation further improves the vaccine by reducing the amount of antigen required to suppress progression of the cancer and tumor growth. Most importantly, the DOTAP/E7-lipopeptide vaccine induced an overall increased CTL activity that is essential for the enhanced tumor clearance and reduced rates of tumor recurrence. This novel formulation represents an excellent candidate for future cancer vaccine development.

\section{Acknowledgment}

We thank Dr. Christine Conwell for her helpful criticism of the manuscript. This work was partially supported by the Mary Kay Ash Charitable Foundation for women cancers. 


\section{Abbreviations Used}

DOTAP, 1,2-dioleoyl-3-trimethylammonium propane; APC, antigen presenting cells; LPD, liposome-polycations-DNA.

\section{References}

1. Connor J, Huang L. pH-sensitive immunoliposomes as an efficient and target-specific carrier for antitumor drugs. Cancer Res 1986;46(7):3431-3435. [PubMed: 2871923]

2. Felnerova D, Viret JF, Gluck R, Moser C. Liposomes and virosomes as delivery systems for antigens, nucleic acids and drugs. Curr. Opin. Biotechnol 2004;15(6):518-529. [PubMed: 15560978]

3. Li SD, Huang L. Targeted delivery of antisense oligodeoxynucleotide and small interference RNA into lung cancer cells. Mol. Pharmaceutics 2006;3(5):579-588.

4. Malik DK, Baboota S, Ahuja A, Hasan S, Ali J. Recent advances in protein and peptide drug delivery systems. Curr. Drug Deliv 2007;4(2):141-151. [PubMed: 17456033]

5. Cui Z, Huang L. Liposome-polycation-DNA (LPD) particle as a carrier and adjuvant for protein-based vaccines: therapeutic effect against cervical cancer. Cancer Immunol. Immunother 2005;54(12):11801190. [PubMed: 15846491]

6. Dileo J, Banerjee R, Whitmore M, Nayak JV, Falo LD Jr. Huang L. Lipid-protamine-DNA-mediated antigen delivery to antigen-presenting cells results in enhanced anti-tumor immune responses. Mol. Ther 2003;7(5 Pt 1):640-648. [PubMed: 12718907]

7. Alving CR. Design and selection of vaccine adjuvants: animal models and human trials. Vaccine 2002;20(3):S56-64. [PubMed: 12184368]

8. Chen WC, Huang L. Non-viral vector as vaccine carrier. Adv. Genet 2005;54:315-337. [PubMed: 16096017]

9. Chen W, Yan W, Huang L. A simple but effective cancer vaccine consisting of an antigen and a cationic lipid. Cancer Immunol. Immunother. 2007in press

10. Yan W, Chen W, Huang L. Mechanism of adjuvant activity of cationic liposome: phosphorylation of a MAP kinase, ERK and induction of chemokines. Mol. Immunol 2007;44(15):3672-3681. [PubMed: 17521728]

11. Gahery-Segard H, Pialoux G, Charmeteau B, Sermet S, Poncelet H, Raux M, Tartar A, Levy JP, GrasMasse H, Guillet JG. Multiepitopic B- and T-cell responses induced in humans by a human immunodeficiency virus type 1 lipopeptide vaccine. J. Virol 2000;74(4):1694-1703. [PubMed: 10644339]

12. Pialoux G, Gahery-Segard H, Sermet S, Poncelet H, Fournier S, Gerard L, Tartar A, Gras-Masse H, Levy JP, Guillet JG. Lipopeptides induce cell-mediated anti-HIV immune responses in seronegative volunteers. AIDS 2001;15(10):1239-1249. [PubMed: 11426068]

13. Livingston BD, Crimi C, Grey H, Ishioka G, Chisari FV, Fikes J, Grey H, Chesnut RW, Sette A. The hepatitis B virus-specific CTL responses induced in humans by lipopeptide vaccination are comparable to those elicited by acute viral infection. J. Immunol 1997;159(3):1383-1392. [PubMed: 9233635]

14. BenMohamed L, Gras-Masse H, Tartar A, Daubersies P, Brahimi K, Bossus M, Thomas A, Druilhe P. Lipopeptide immunization without adjuvant induces potent and long-lasting B, T helper, and cytotoxic T lymphocyte responses against a malaria liver stage antigen in mice and chimpanzees. Eur. J. Immunol 1997;27(5):1242-1253. [PubMed: 9174617]

15. Kanafani ZA, Corey GR. Daptomycin: a rapidly bactericidal lipopeptide for the treatment of Grampositive infections. Expert Rev. Anti Infect. Ther 2007;5(2):177-184. [PubMed: 17402833]

16. Denning DW, Marr KA, Lau WM, Facklam DP, Ratanatharathorn V, Becker C, Ullmann AJ, Seibel NL, Flynn PM, van Burik JA, Buell DN, Patterson TF. Micafungin (FK463), alone or in combination with other systemic antifungal agents, for the treatment of acute invasive aspergillosis. J. Infect 2006;53(5):337-349. [PubMed: 16678903]

17. Byers AM, Kemball CC, Moser JM, Lukacher AE. Cutting edge: rapid in vivo CTL activity by polyoma virus-specific effector and memory CD8+ T cells. J. Immunol 2003;171(1):17-21. [PubMed: 12816977] 
18. Melief CJ. Tumor eradication by adoptive transfer of cytotoxic T lymphocytes. Adv. Cancer Res 1992;58:143-175. [PubMed: 1532109]

19. Melief CJ, Van Der Burg SH, Toes RE, Ossendorp F, Offringa R. Effective therapeutic anticancer vaccines based on precision guiding of cytolytic T lymphocytes. Immunol. Rev 2002;188:177-182. [PubMed: 12445291]

20. Feltkamp MC, Smits HL, Vierboom MP, Minnaar RP, de Jongh BM, Drijfhout JW, ter Schegget J, Melief CJ, Kast WM. Vaccination with cytotoxic T lymphocyte epitope-containing peptide protects against a tumor induced by human papillomavirus type 16-transformed cells. Eur. J. Immunol 1993;23(9):2242-2249. [PubMed: 7690326]

21. Fernandes I, Frisch B, Muller S, Schuber F. Synthetic lipopeptides incorporated in liposomes: in vitro stimulation of the proliferation of murine splenocytes and in vivo induction of an immune response against a peptide antigen. Mol. Immunol 1997;34(8-9):569-576. [PubMed: 9393959]

22. Yagi N, Ogawa Y, Kodaka M, Okada T, Tomohiro T, Konakahara T, Okuno H. Preparation of functional liposomes with peptide ligands and their binding to cell membranes. Lipids 2000;35(6): 673-680. [PubMed: 10901430]

23. Liang MT, Davies NM, Toth I. Encapsulation of lipopeptides within liposomes: effect of number of lipid chains, chain length and method of liposome preparation. Int. J. Pharm 2005;301(1-2):247254. [PubMed: 16054787]

24. Verheul AF, Udhayakumar V, Jue DL, Wohlhueter RM, Lal AA. Monopalmitic acid-peptide conjugates induce cytotoxic $\mathrm{T}$ cell responses against malarial epitopes: importance of spacer amino acids. J. Immunol. Methods 1995;182(2):219-226. [PubMed: 7540640]

25. Schild H, Deres K, Wiesmuller KH, Jung G, Rammensee HG. Efficiency of peptides and lipopeptides for in vivo priming of virus-specific cytotoxic T cells. Eur. J. Immunol 1991;21(11):2649-2654. [PubMed: 1936113]

26. Wiesmuller KH, Bessler WG, Jung G. Solid phase peptide synthesis of lipopeptide vaccines eliciting epitope-specific B-, T-helper and T-killer cell response. Int. J. Pept. Protein Res 1992;40(3-4):255260. [PubMed: 1282504]

27. da Fonseca DP, Joosten D, van der Zee R, Jue DL, Singh M, Vordermeier HM, Snippe H, Verheul AF. Identification of new cytotoxic T-cell epitopes on the 38-kilodalton lipoglyco-protein of Mycobacterium tuberculosis by using lipopeptides. Infect. Immun 1998;66(7):3190-3197. [PubMed: 9632585]

28. Ljunggren HG, Stam NJ, Ohlen C, Neefjes JJ, Hoglund P, Heemels MT, Bastin J, Schumacher TN, Townsend A, Karre K. Empty MHC class I molecules come out in the cold. Nature 1990;346(6283): 476-480. [PubMed: 2198471] 


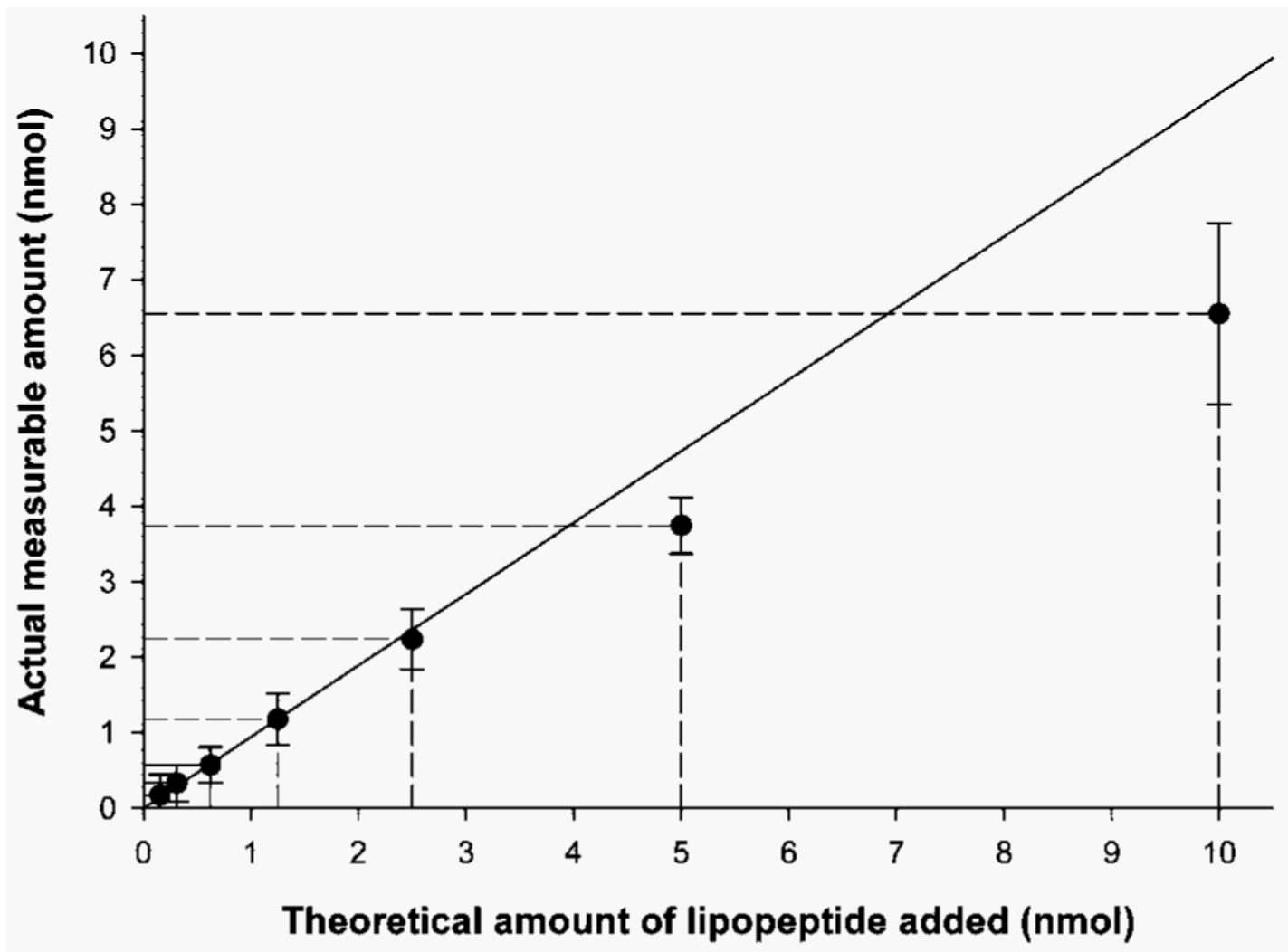

Figure 1.

Entrapment efficiency of the E7-lipopeptide in DOTAP liposome. Lipopeptide varying from 0.16 to $10 \mathrm{nmol}$ was mixed with DOTAP lipid of $100 \mathrm{nmol}$, and the peptide entrapment efficiency was measured after liposome preparation. The solid line shows a regression curve with a slope equal to $95 \%$. 


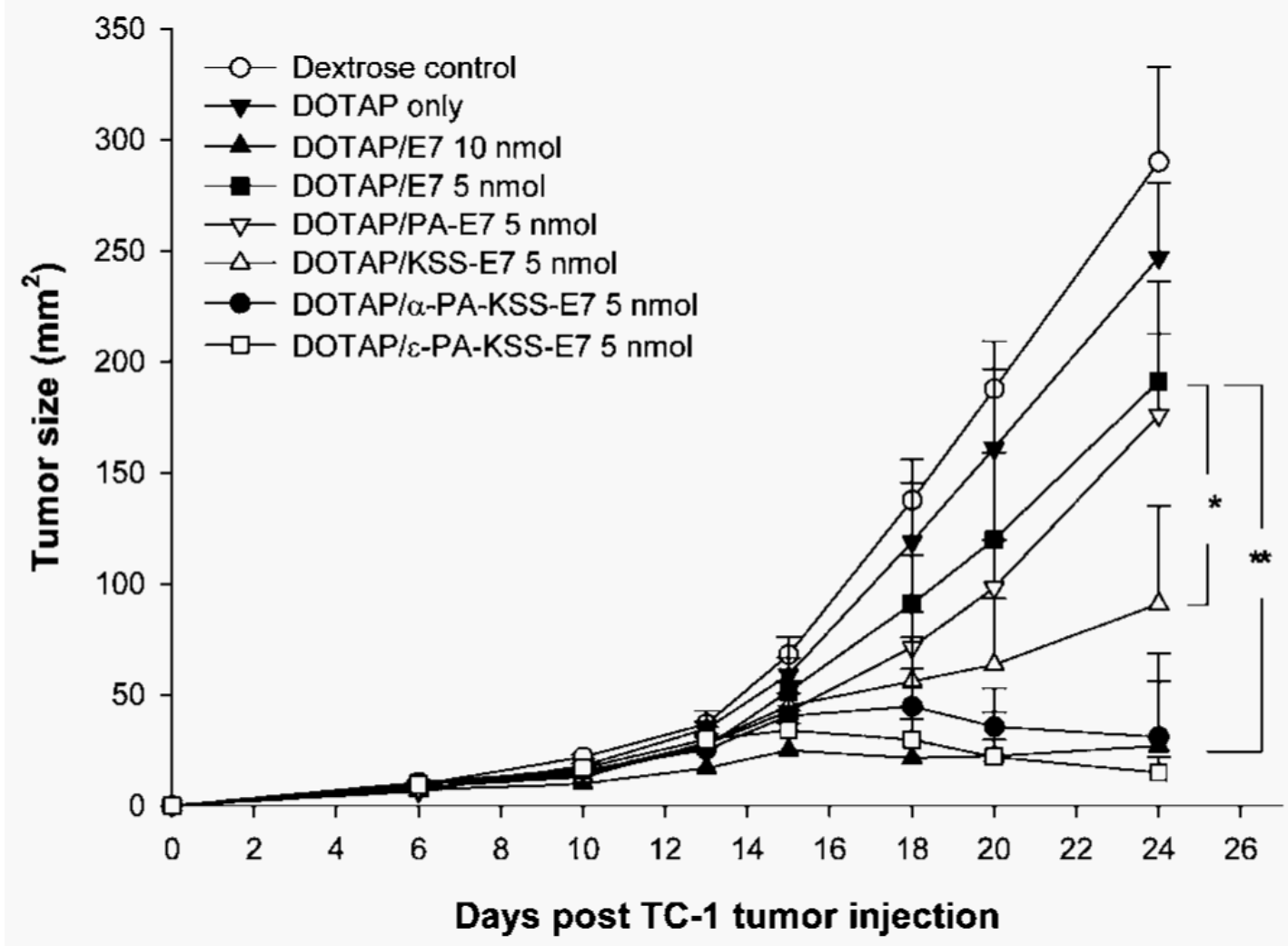

Figure 2.

Anti TC-1 tumor activity was enhanced by incorporation of E7-lipopeptide in the cationic liposomal formulation. TC-1 tumor bearing mice (8-12 mice per group) received a single treatment on day 6 with DOTAP/E7 (containing E7 peptide of 5 or $10 \mathrm{nmol}$ ) or DOTAP/E7lipopeptide (containing $5 \mathrm{nmol}$ of peptide) or DOTAP lipid alone. Mice treated with dextrose $(5 \%)$ were used as a negative control. Tumor size was determined by multiplying the two largest dimensions of the solid tumor. The mean of tumor sizes of each group at day 24 was compared to that of the group which was received DOTAP/E7 $(5 \mathrm{nmol})$ and was analyzed statistically $(* p<0.05, * * p<0.01)$. 

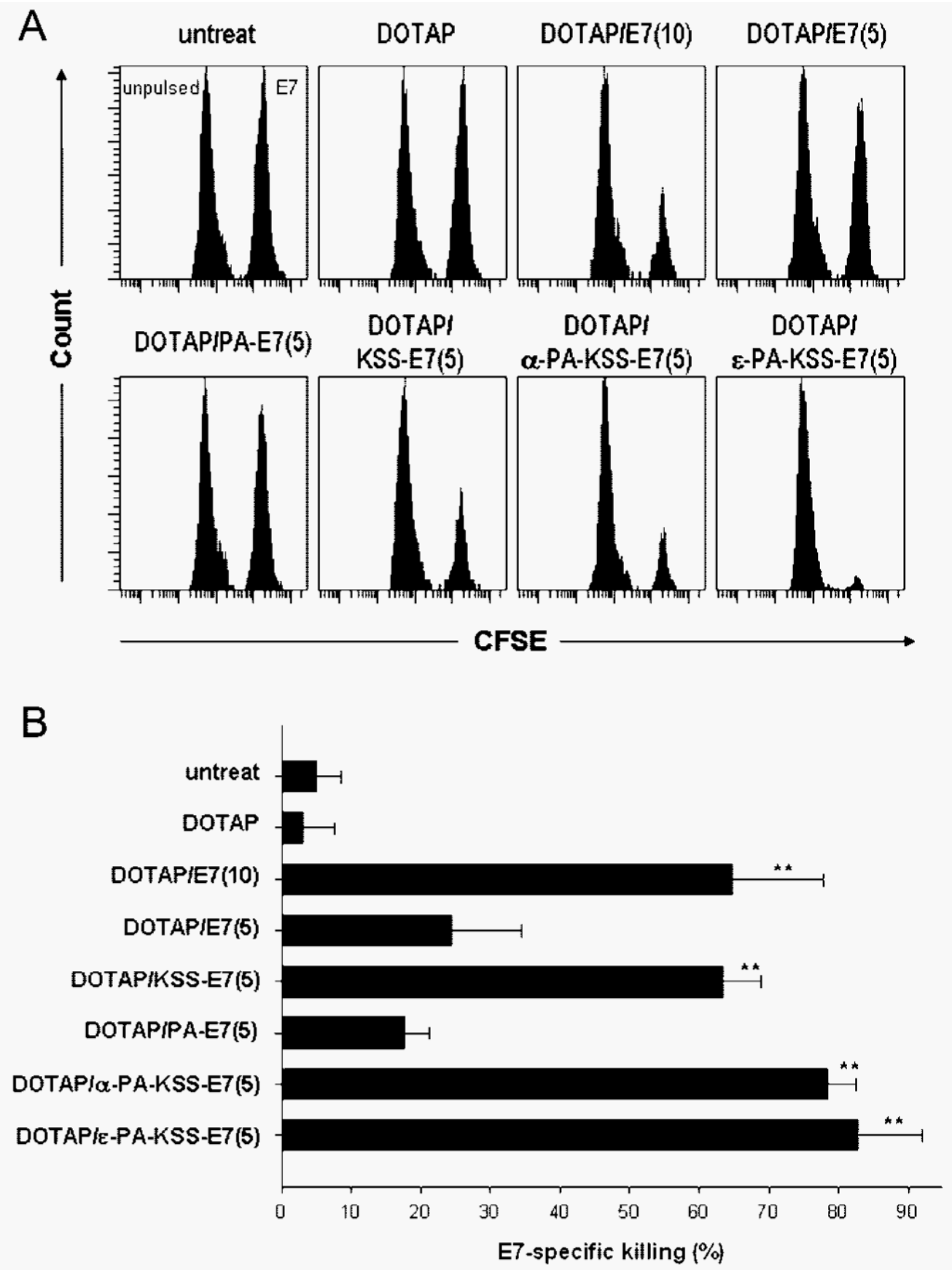

Figure 3.

Immunization with DOTAP/E7-lipopeptide formulation elicited an enhanced in vivo CTL response. (A) C57BL/6 mice were s.c. immunized with DOTAP/E7 (containing E7 peptide of 5 or $10 \mathrm{nmol})$, DOTAP/KSS-E7 (5 nmol), DOTAP/E7-lipopeptide (5 nmol), or DOTAP lipid alone at day 0 and day 10 . Mice left untreated were used as a negative control. Seven days after the last immunization, representative mice were i.v. injected with an equivalent amount of E7pulsed (labeled with $4 \mu \mathrm{M}$ CFSE) and nonpulse (labeled with $0.4 \mu \mathrm{M}$ CFSE) spleen cells obtained from a syngenic donor. After $16 \mathrm{~h}$, spleen cells from the adoptively transferred mice were harvested, and the proportions of the CFSE ${ }^{\text {high }}$ and $\mathrm{CFSE}^{\text {low }}$ cells were analyzed by flow cytometry. (B) Percentage of specific anti-E7 killing was shown. The mean of the percentage 
from each group was compared to that of the group received DOTAP/E7 $(5 \mathrm{nmol})$ and was analyzed statistically $(* * p<0.01, n) 4)$. 


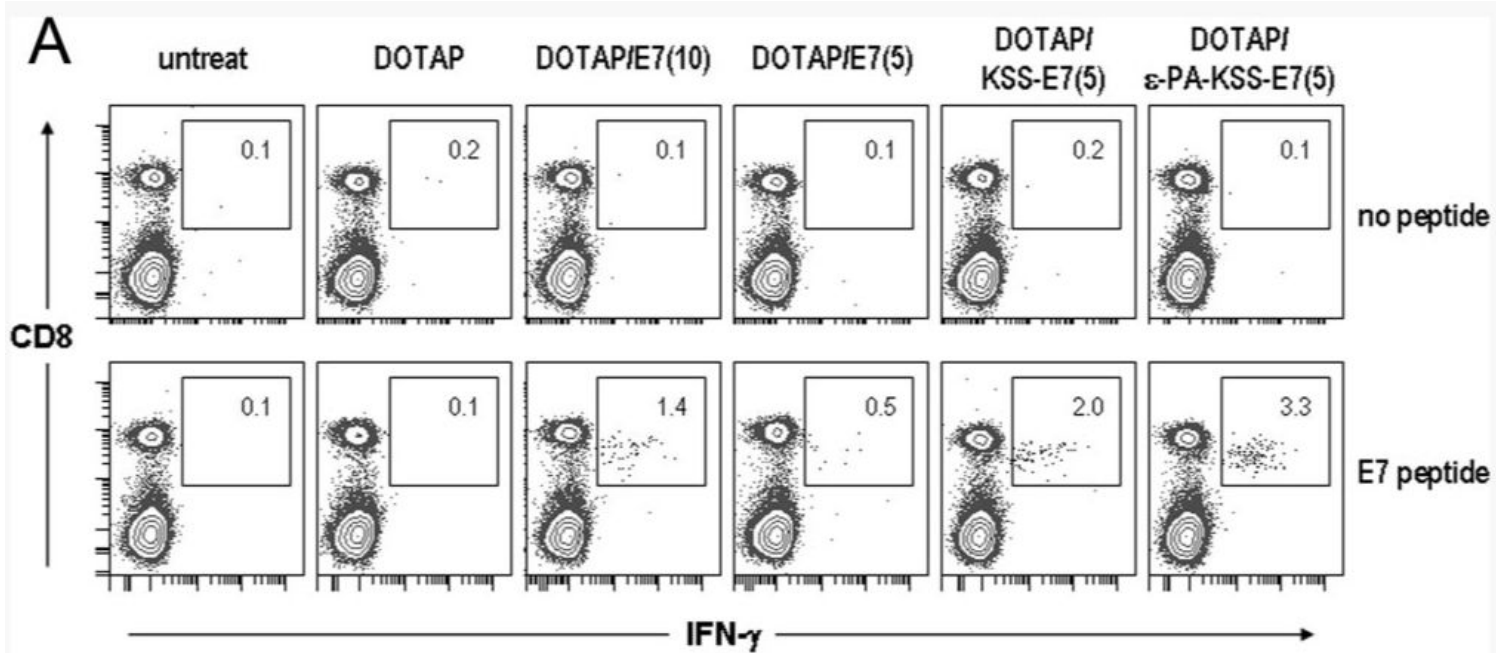

B

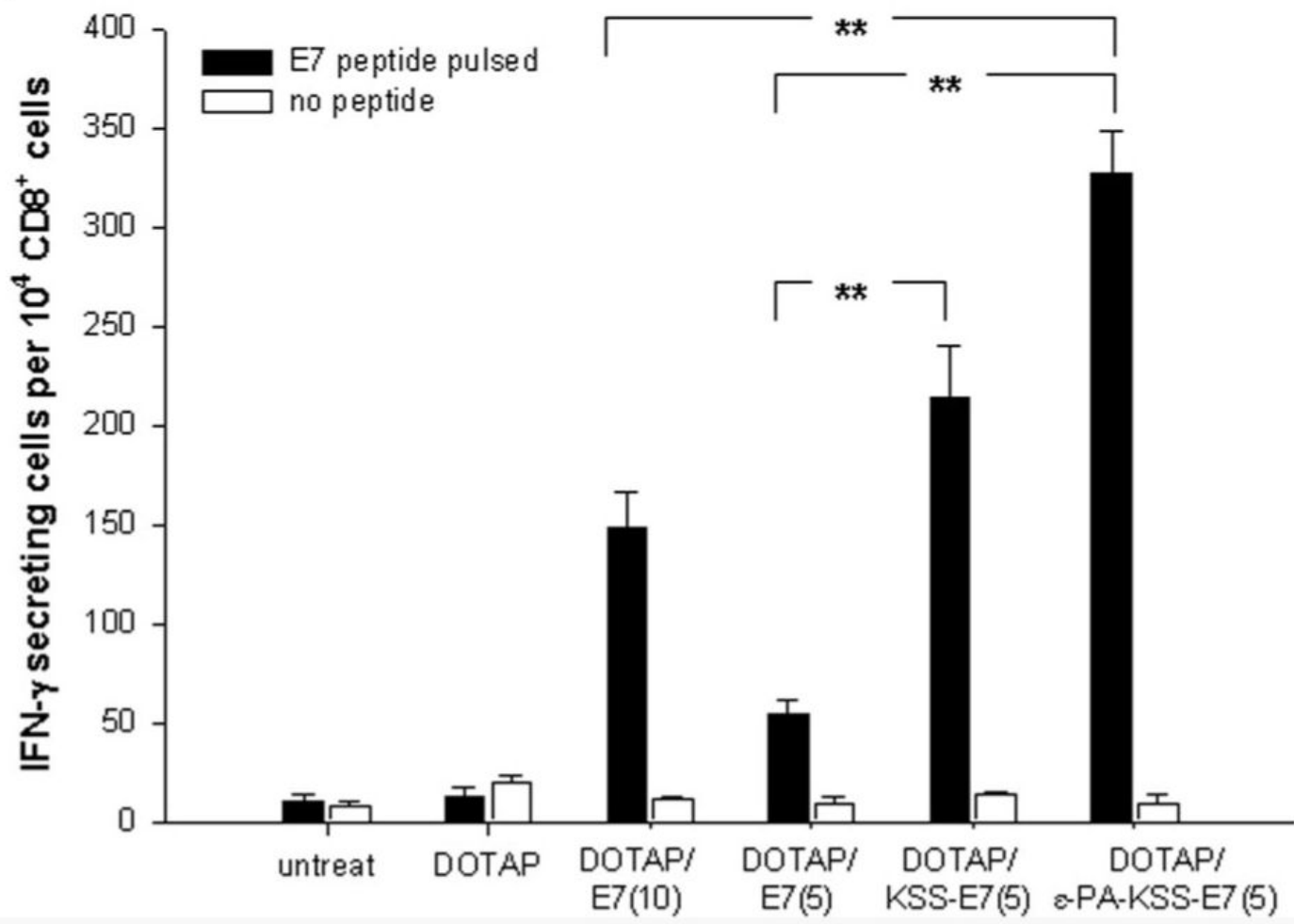

Figure 4.

Immunization with DOTAP/E7-lipopeptide increased the production of IFN- $\gamma$ secreting $\mathrm{CD}^{+} \mathrm{T}$ cells. (A) Mice were immunized as described, and 7 days after the last immunization, spleen cells from immunized mice were isolated. The cells were stimulated in vitro with or without E7 peptide $(5 \mu \mathrm{g} / \mathrm{mL})$ for $6 \mathrm{~h}$ and were stained with a surface CD8 marker and an intracellular IFN- $\gamma$ cytokine prior to FACS analysis. The numbers shown on contour plots represent the percentages of $\mathrm{CD}^{+}{ }^{+} \mathrm{IFN}-\gamma^{+} \mathrm{T}$ cells gated on the total $\mathrm{CD} 8^{+}$cells. Representative figures in four experiments performed. (B) The percentage of CD $8^{+} \mathrm{IFN}-\gamma^{+}$double-positive cells per $10^{4}$ total $\mathrm{CD}^{+}$from each treatment group were shown as mean $\pm \mathrm{SD}$ and were statistically analyzed by paired $t$ test $(* * p<0.01, n=4)$. 


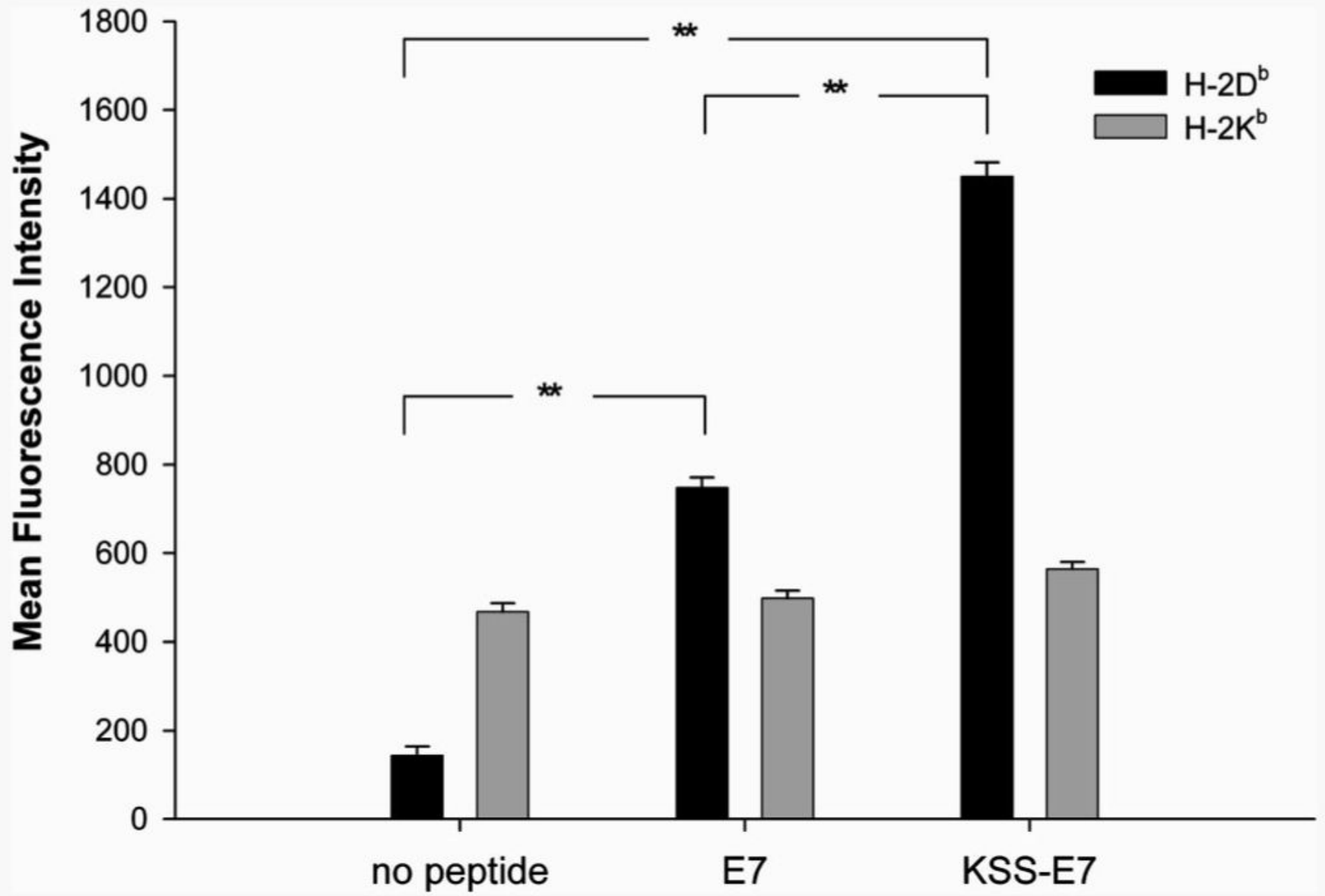

Figure 5.

MHC class I molecules up-regulation by E7 and KSS-E7 peptide. RMA-S cells were incubated with E7 or KSS-E7 peptide at $10 \mu \mathrm{M}$, and the up-regulation of MHC class I molecules were detected by a fluorescently conjugated $m A b$ against $\mathrm{H}-2 \mathrm{D}^{\mathrm{b}}$ or $\mathrm{H}-2 \mathrm{~K}^{\mathrm{b}}$. Cells treated with medium were used as a control. The results are expressed as mean fluorescence intensity of triplicate determination $\pm \mathrm{SD}$ and were statistically analyzed by paired $t$ test $(* * p<0.01, n=$ $3)$. 


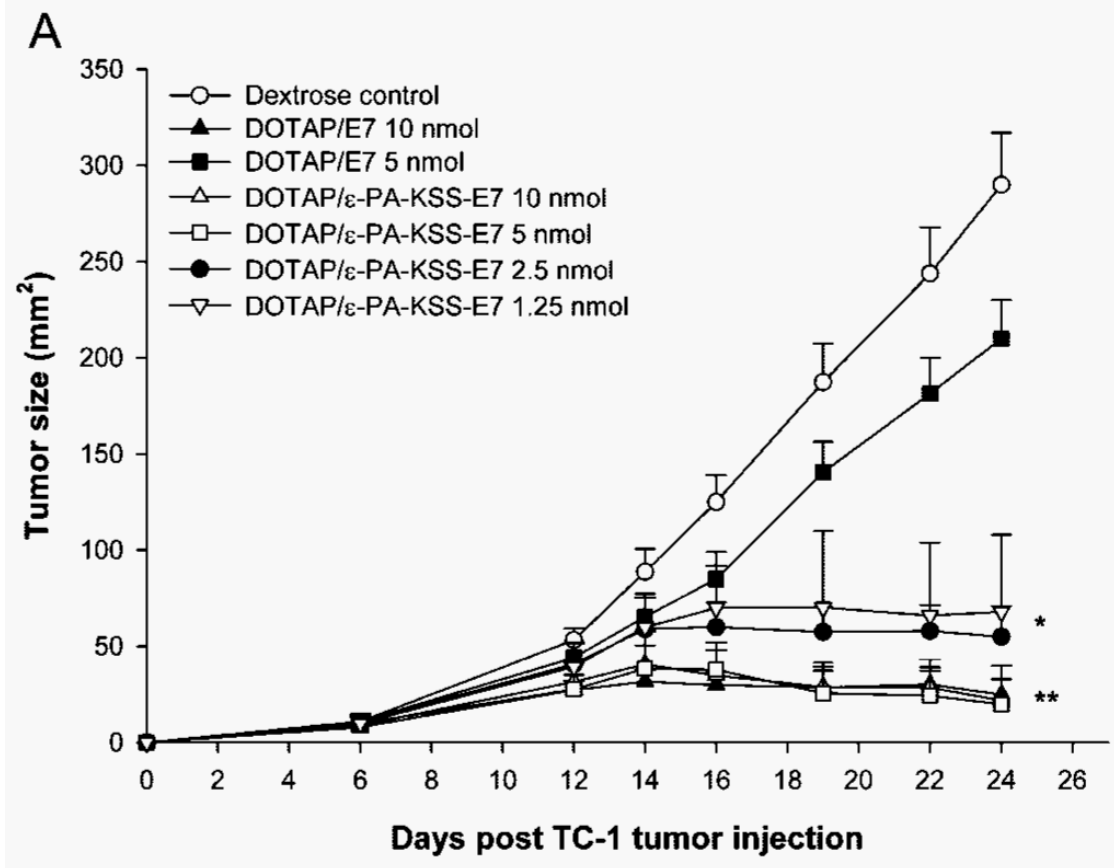

B

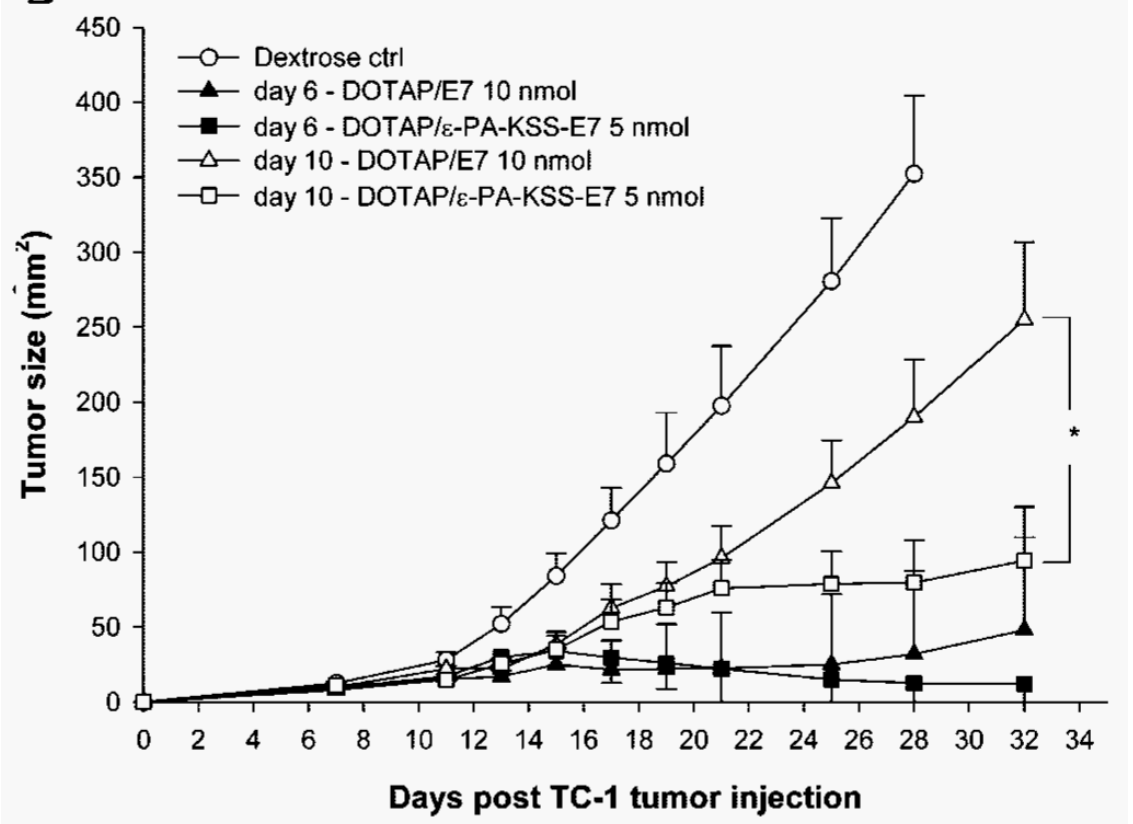

Figure 6.

Potency of DOTAP/E7-lipopeptide vaccine for the treatment of TC-1 tumor. (A) Tumor bearing mice were established as described and were given a single treatment of DOTAP/E7 or DOTAP/ $\epsilon$ PA-KSS-E7 formulation on day 6. Lipopeptides varying from 1.25 to $10 \mathrm{nmol}$ per dose were investigated. The mean of the tumor sizes from each group at day 24 was compared to that of the group receiving DOTAP/E7 $(5 \mathrm{nmol})$ and was analyzed by one-way ANOVA $(* p<0.05, * * p<0.01, n=6)$. (B) TC-1 tumor-bearing mice were treated with DOTAP/E7 (10 nmol) or DOTAP/ $\epsilon$ PA-KSS-E7 $(5 \mathrm{nmol})$ formulation on day 6 (solid 
symbols) or day 10 (open symbols). The mean of the tumor sizes at day 32 was compared between the two formulations $\left({ }^{*} p<0.05, n=6\right)$. 


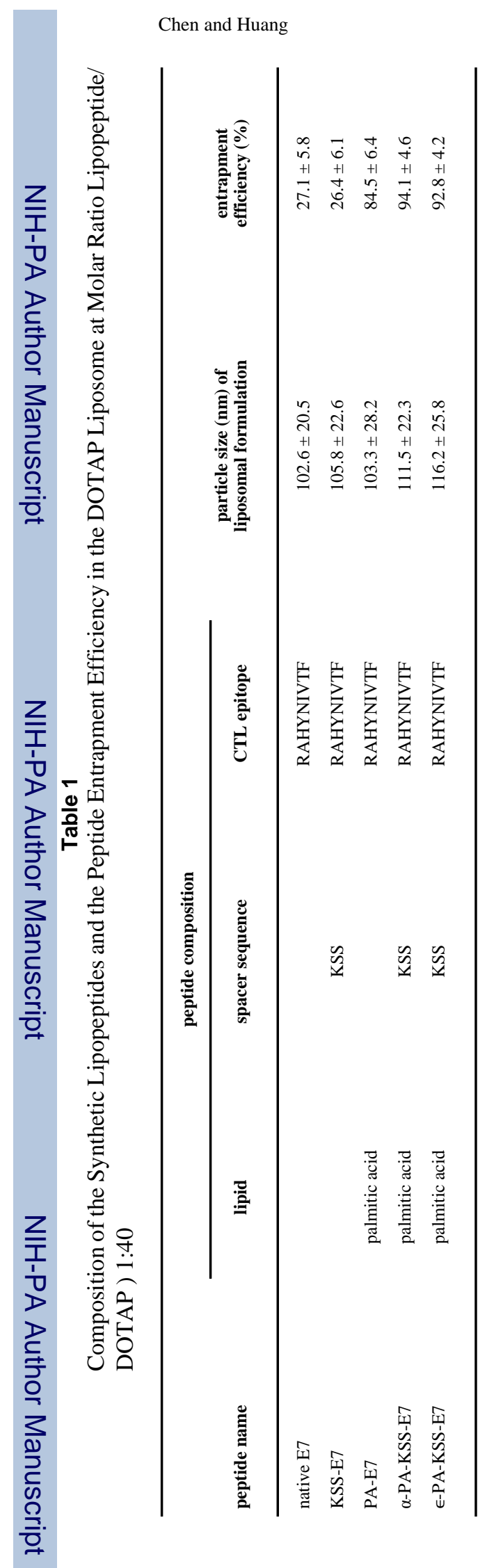

Page 18 